\title{
Applications of Eccentric Exercise to Improve Muscle and Mobility Function in Older Adults
}

\author{
Dae Young Kim ${ }^{1,2}$, Seung Lyul Oh ${ }^{1,3}$, Jae-Young Lim ${ }^{1,3}$ \\ ${ }^{1}$ Department of Rehabilitation Medicine, Aging and Mobility Biophysics Laboratory, Seoul National University Bundang Hospital, Seongnam, Korea \\ ${ }^{2}$ Health and Exercise Science Laboratory, Institute of Sports Science, Seoul National University, Seoul, Korea \\ ${ }^{3}$ Institute on Aging, Seoul National University, Seoul, Korea
}

\section{Corresponding Author:}

Jae-Young Lim, MD, PhD

Department of Rehabilitation Medicine,

Aging and Mobility Biophysics

Laboratory, Seoul National University

Bundang Hospital, 82 Gumi-ro

173beon-gil, Bundang-gu, Seongnam

13620, Korea

E-mail: drlim1@snu.ac.kr

ORCID:

https://orcid.org/0000-0002-9454-0344

Received: December 7, 2021

Accepted: January 11, 2022
Muscle aging ultimately leads to the deterioration of human physiological functioning, including declining muscle strength, loss of muscle mass, and decreased quality of life in advanced age. Eccentric exercise is a key intervention that has the potential to ameliorate this problem. Recent studies have focused on evidence-based exercise interventions to prevent declines in muscle strength and physical function in older adults. This paper reviewed relevant literature on the use of eccentric exercise to improve muscle and mobility function in older adults. We explained not only the changes in mobility that occur with aging but also the rationale for and positive effects of eccentric intervention in older adults. We also explored several proposed mechanisms for the intramuscular changes caused by eccentric muscle contraction and considered the safety and side effects accompanying eccentric training. We concluded by suggesting that eccentric exercise is an exercise modality that can potentially improve muscle strength and enhance mobility in older adults.

Key Words: Aging, Exercise, Mobility, Muscles

\section{INTRODUCTION}

Aging is accompanied by a series of adverse changes, typically including the loss of $30 \%-40 \%$ of skeletal muscle mass, particularly type II fibers. ${ }^{1)}$ The muscle mass at $60-70$ years of age is approximately $70 \%-80 \%$ that in younger people ( $<60$ years old) and is also accompanied by diminished muscle strength. ${ }^{2)}$ Certain characteristics commonly observed in older adults reflect limitations due to diminished muscular strength and mobility functions. ${ }^{3,4)}$ Therefore, it is essential to develop exercise interventions that maintain mobility by counteracting the decline of muscular and mobility functions and improving the quality of life of older adults. ${ }^{5)}$ However, it is difficult to apply conventional resistance exercises, which largely comprise isometric and concentric contractions, at sufficient intensity and amount to increase strength and improve function in older individuals with limited physical func- tion and mobility. ${ }^{6,7)}$ Thus, mechanism-based specific modalities have received attention; among these are the eccentric-based mod$\mathrm{el}$, which we will introduce.

Eccentric exercise has been introduced as a specific method to improve muscle strength and mobility in older adults. ${ }^{5,8)}$ Eccentric muscle contractions occur when an active muscle force increases substantially during lengthening. ${ }^{9,10)}$ Eccentric or lengthening contractions often occur during routine daily activities, such as descending stairs or transitioning from standing to sitting. ${ }^{11)}$ Training programs primarily employing repetitive sub-maximal eccentric muscle contractions are a common form of exercise and an effective mode of conditioning for improving muscular strength and mobility in older adults. However, some have argued that resistance exercise can increase the risk of injury, reduce participation, and lead to boredom due to extended repetition. ${ }^{12)}$ Thus, it is essential to create specific exercise interventions that 
are effective at preventing or delaying functional decline based on age-related changes in the muscle and mobility systems of older adults.

We reviewed the literature on the effectiveness, appropriate protocols, and relevant mechanisms of eccentric exercise for the improvement of muscle and mobility function in older adults, especially in comparison with other forms of traditional resistance exercise.

\section{EFFECTS OF AGING ON MOBILITY AND SKELETAL MUSCLE FUNCTION}

Age-related sarcopenia is a geriatric syndrome characterized by the loss of skeletal muscle mass and strength, which can severely limit muscle function. ${ }^{13-15)}$ The main consequences of these age-related changes are functional limitation, disability, increased susceptibility to injuries, and increased morbidity and mortality. ${ }^{16,17)}$ The overall decline in functional independence may seriously compromise the quality of life of older adults. The mobility function is closely correlated with changes in the muscle force-velocity relationship, ${ }^{18)}$ and a slower walking speed is one of the representative changes linked to functional limitations. ${ }^{8)}$ The major changes reported in aging skeletal muscles observed in older adults include decreased muscle fiber number and muscle cross-sectional area, as well as defective regeneration. ${ }^{19)}$ Fiber-type changes constitute one reported mechanism by which muscle function is lost with aging, with type II fibers being more susceptible to atrophy than type I fibers. ${ }^{20)}$ Furthermore, specific changes in the intrinsic ability of aging muscles to generate force have also been observed. ${ }^{21)}$ The intrinsic contractile characteristics related to the cross-bridge mechanics of single fibers are altered with aging. ${ }^{22)}$ Both decreased contractile tissues and reduced force-generating capacity per cross-bridge affect the contractile properties of aging muscles. ${ }^{8,22,23)}$ A decrease in the specific force (normalized force per cross-sectional area) and unloaded shortening velocity of type I and IIa fibers in older adults compared to younger adults have also been reported. ${ }^{24,25)}$ These findings provide evidence that both intrinsic changes in muscle quality and a loss of muscle mass contribute to age-associated changes in skeletal muscle function.

To prevent age-related decline in physical function, exercise interventions that maintain or increase muscle mass and muscle quality are primarily considered. Regular resistance exercises have been shown to counteract most aspects of physiological deterioration due to aging. ${ }^{26,27)}$ However, older individuals with limited ability to perform exercises may have difficulty participating in conventional exercise interventions and, even if they do participate, are often unable to exercise sufficiently for the intervention to be effective. ${ }^{8)}$ Therefore, exercise interventions or modalities specific to age-related changes and functional limitations are required for mobility-impaired older populations.

\section{ECCENTRIC EXERCISE AND ITS APPLICATION IN OLDER ADULTS}

Traditional resistance exercises, which emphasize concentric contraction, are effective in improving muscle function and restoring physical function. However, they are limited as an exercise method in the context of the physiological changes accompanying advancing age. ${ }^{28)}$ For older adults, exercises must provide substantial changes in the intrinsic properties of skeletal muscles to improve the performance of functional movements in daily life. ${ }^{29)}$ To overcome the limitations of conventional exercise interventions, a more specific intervention strategy targeting the changing physiological characteristics and muscular function associated with aging is warranted. Among various intervention strategies, eccentrically-biased exercise, especially chronic eccentric exercise, is reportedly superior to conventional training in terms of increasing muscle strength and function in aging populations. ${ }^{30)}$ As the interest in specific modalities to counteract age-related muscle atrophy or functional decline has increased, various types of interventions focusing on eccentric contraction have been applied to older populations in clinical and community-based settings. ${ }^{8)}$ Thus, it is necessary to review the current and relevant literature on the effectiveness of eccentric exercise modalities, especially in terms of their comparative effectiveness to other modalities, specific protocols, and intervention settings, including the equipment or tools used in the interventions.

This review searched the PubMed, Medline, Embase, and Cochrane Library databases in January 2021, using the following keywords: older adults, aging, eccentric exercise, eccentric contraction, muscle strength, and physical function. Based on the titles and some parts of the articles when needed, we screened the literature to select articles that met the following inclusion criteria: (1) participants aged 60 years or older, (2) randomized controlled tri$\mathrm{al}$, and (3) at least one group that performed eccentric exercise. We restricted our review to studies in English, owing to the translation of non-English language studies and lack of resources for review. The primary database search yielded 111 articles. After removing duplicates, 92 articles were considered potentially relevant and screened for content. Among them, 67 articles were excluded based on the training characteristics and study objectives. Of the 25 studies reviewed, 10 met our predetermined criteria (Fig. 1). Detailed information from each article, including author, year of 


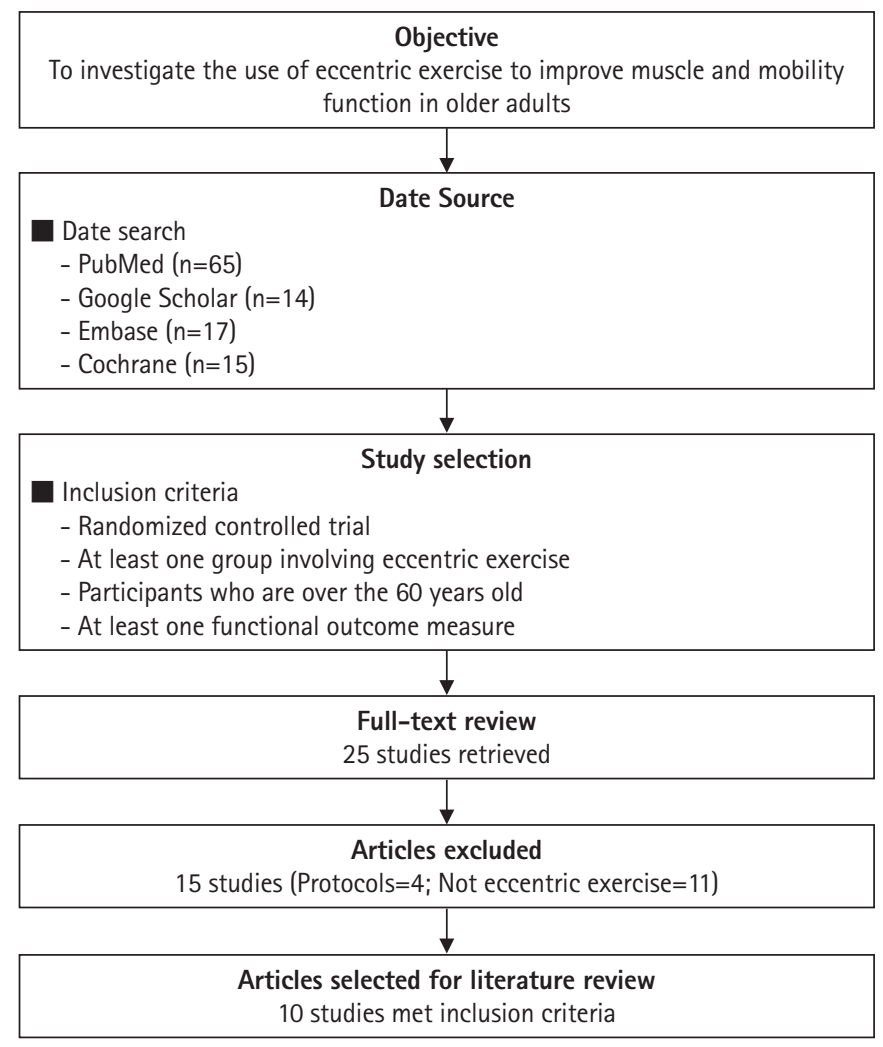

Fig. 1. Flowchart of the methodology used in this review.

publication, study design, and exercise characteristics (type, intensity, frequency, and duration) is shown in Table 1. As these studies employed different eccentric exercise programs and protocol treatments, it is difficult to determine whether the effects observed were solely due to eccentric exercise.

Some studies ${ }^{31-35)}$ used specialized equipment, such as eccentric ergometers, isokinetic dynamometers, and flywheel ergometers. Kim et al., ${ }^{31)}$ Mueller et al., ${ }^{33)}$ and LaStayo et al. ${ }^{32)}$ used eccentric ergometers for eccentric training. Mueller et al. ${ }^{33)}$ reported a significant increase in isometric strength. Isokinetic strength and power were also significantly increased following training using an eccentric ergometer. ${ }^{31)}$ However, LaStayo et al..$^{32}$ reported no significant differences between traditional resistance and eccentric groups in the fall risk variables of mobility and leg extensor muscle power. However, as this study did not employ power training in the eccentric or conventional resistance exercise, it would be hard to expect significant changes in variables related to power output. Squatbased exercise interventions using a flywheel in the study by Sanudo et al. resulted in significant increases in balance and power in older participants. ${ }^{35)}$ Raj et al..$^{34}$ employed eccentric exercises using an isokinetic dynamometer, which resulted in significant improvement in maximum one-repetition maximum (1RM) exercises and isometric and isokinetic torque. Collectively, these results indicated that the use of specialized equipment for eccentric contraction provided significant benefits in muscle and mobility function in older adults.

Other than interventions with specialized equipment, other studies have applied various types of eccentric contraction protocols. ${ }^{36-40)}$ Eccentric exercise interventions, such as regular body squats without a device and the use of a leg extension machine, have been performed in older adults. ${ }^{40,41)}$ These studies showed significant improvements in isometric, eccentric, and concentric peak torque, demonstrating that basic manual resistance exercise training emphasizing eccentric muscle contractions could improve muscle strength and physical function. Dias et al. ${ }^{36)}$ reported on the application of eccentric (concentric and eccentric phases set at 1.5 and 4.5 seconds, respectively) and conventional resistance (concentric and eccentric phases set at 1.5 seconds training) in older women. Both eccentric and concentric training improved knee extension at 1RM, the Timed Up-and-Go test, and the 6-meter walk test. ${ }^{36)}$ Other studies reported that the effect of eccentric exercise was not superior to that of conventional resistance exercise, and some outcomes indicated no effects. Eccentrically-biased training using a leg press resulted in no significant improvements in leg extension strength, leg curl strength, or 1RM maximal strength. ${ }^{37,38)}$ This finding probably indicated insufficient training to induce adaptation or that the adaptation failed to affect muscle strength or functional ability.

These results indicated that pure eccentric exercise using specialized equipment might improve overall muscle strength to a greater extent than eccentrically-biased or combined eccentric and concentric contraction ${ }^{31,42)}$ and that this treatment modality can overload the muscular system at a low energy cost. ${ }^{43)}$ Therefore, eccentric exercise can produce relatively high intensity with low volume, making it an attractive exercise option in strength programs to improve physical performance in older adults. ${ }^{44)}$

\section{THE BENEFITS OF ECCENTRIC CONTRACTION IN OLDER ADULTS}

Eccentric exercise consists mainly of eccentric isotonic contractions, wherein the muscle is forcefully lengthened. Eccentric exercise has various benefits in older adults. Eccentric contraction facilitates higher force production compared to other types of resistance exercises. ${ }^{31}$ The relative preservation of eccentric strength in older adults compared to isometric and concentric strength is well-established (Fig. 2). This reserve of eccentric strength could be beneficial in resistance training programs for people with reduced levels of muscle force. ${ }^{45)}$ Eccentric contraction also tends to entail higher contraction velocities and reduced dependent con- 


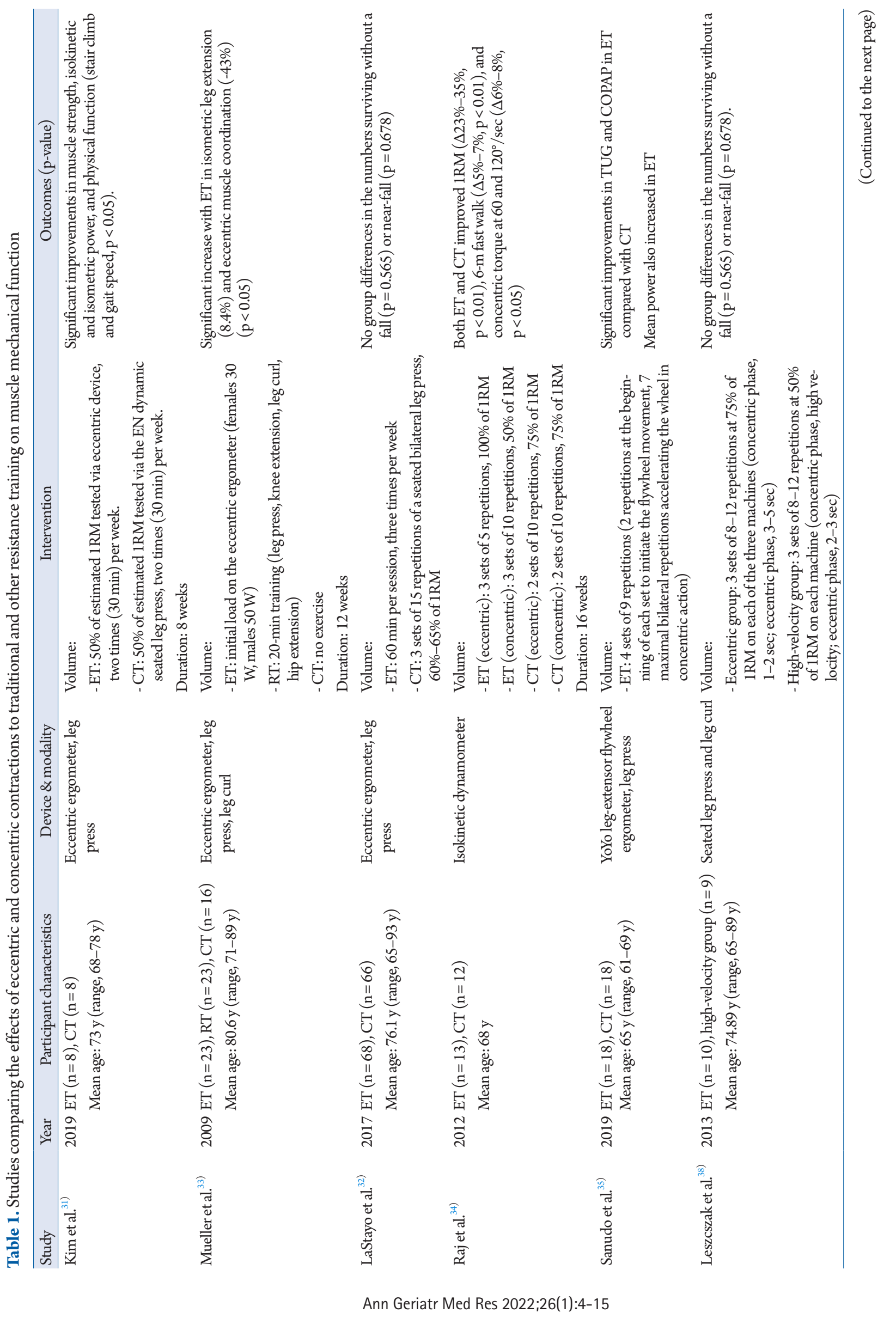




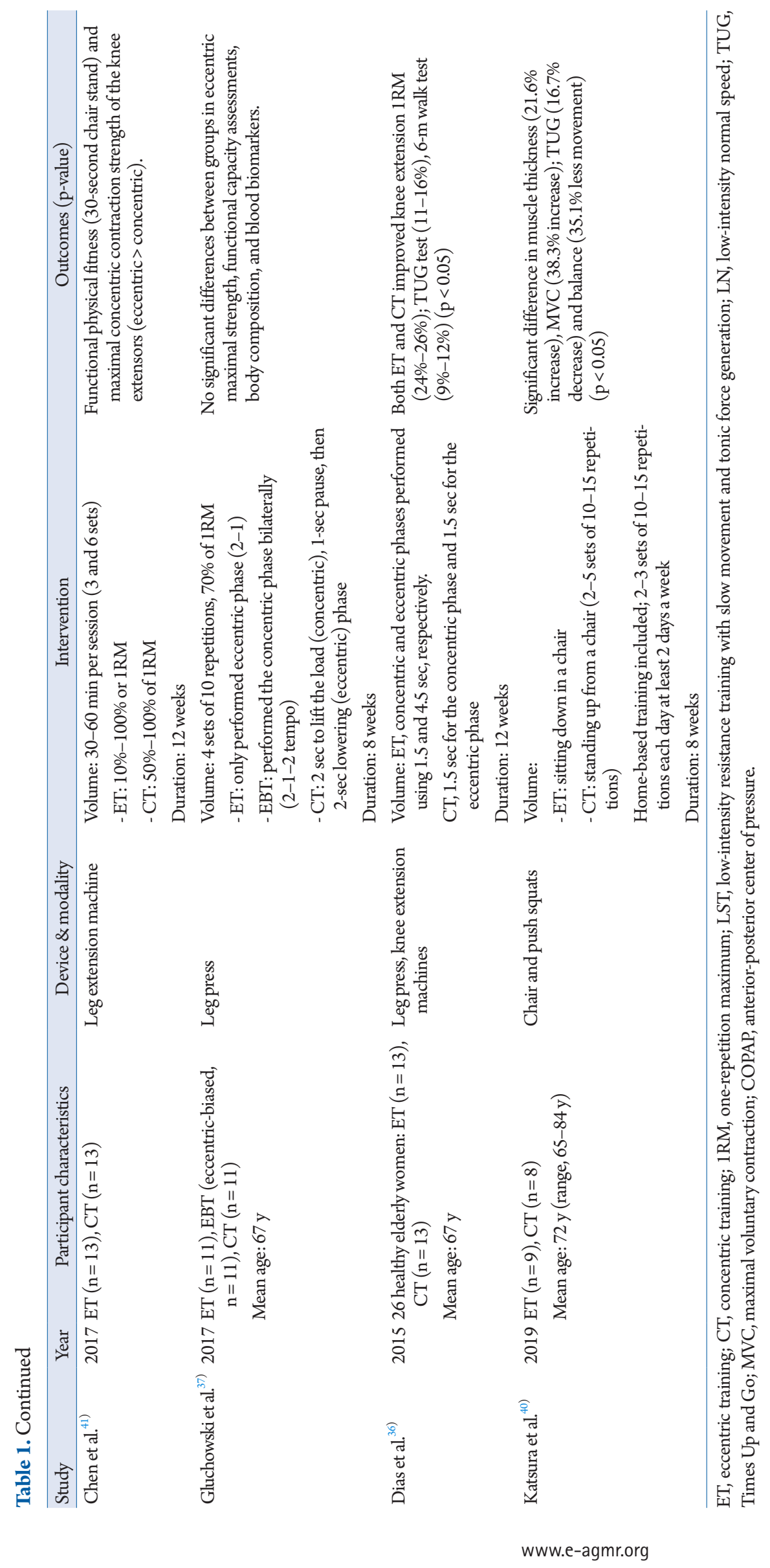



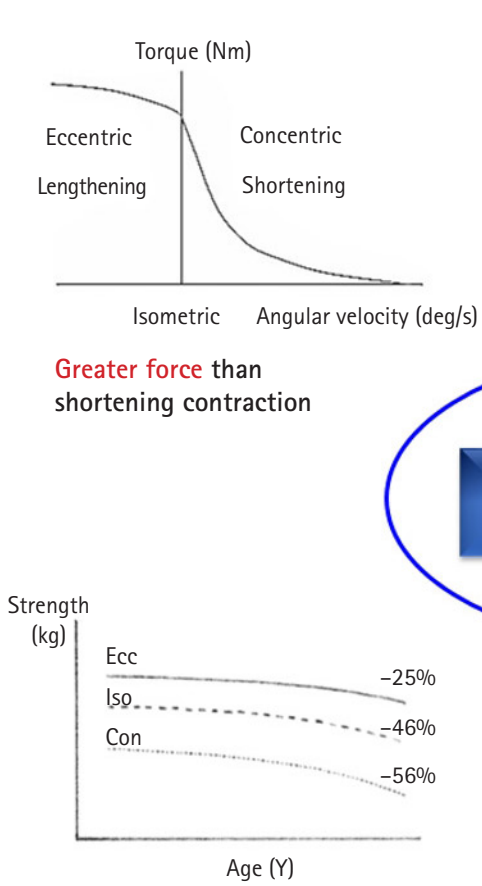

Relative preservation of eccentric strength

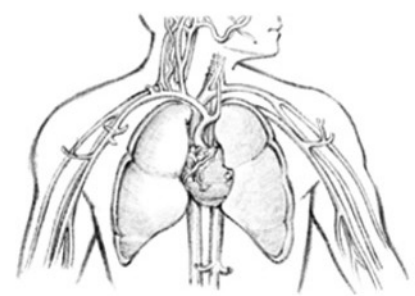

Lower metabolic cost and cardiorespiratory response

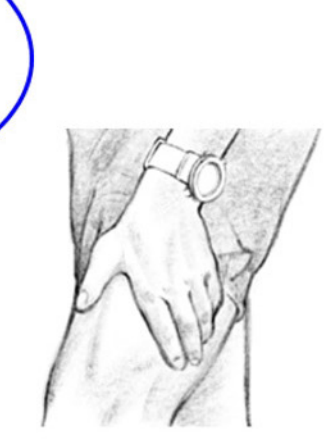

Less DOMS in the older group
Fig. 2. Advantages of eccentric contraction in older adults. DOMS, delayed onset of muscle soreness. traction velocities. ${ }^{46)}$

Another advantage of eccentric exercise compared to concentric exercise for older adults is the lower metabolic cost and cardiorespiratory burden for the same amount of exercise, which enables efficient muscle strengthening with low energy consumption. ${ }^{6,47,48)}$ The low metabolic cost and minimal cardiorespiratory burden imposed by eccentric exercise could be a therapeutic advantage for older adults, who are often vulnerable to metabolic demands. Thus, chronic eccentric exercise may be effectively used by older people who are functionally limited because high energy consumption is not required.

Delayed onset of muscle soreness (DOMS) is a known risk factor that interferes with exercise continuation (Fig. 2). DOMS includes pain, swelling, tenderness, and stiffness due to extracellular matrix damage, impaired sarcolemma permeability, and ultrastructural damage induced by eccentric contraction. However, this phenomenon occurs less frequently in older adults, mainly due to the characteristics of their muscles, in which type II fibers are predominately reduced. ${ }^{49)}$ Additionally, high-intensity or high-volume eccentric exercise results in increased cross-sectional area of type II muscle fibers, ${ }^{50,51)}$ as well as a shift toward fast type II muscle phenotypes. ${ }^{52)}$ Therefore, eccentric exercise is considered an exercise intervention strategy that reflects the physiological characteristics of older individuals and is expected to be safe and effective.

\section{MECHANISMS OF MUSCLE FUNCTION IMPROVEMENT BY ECCENTRIC EXERCISE}

Previous experimental studies have proposed mechanisms to explain muscle quality change following eccentric muscle contractions. ${ }^{53-60)}$ Detailed information, including subject characteristics, exercise device and modality, and type of intervention in these studies, are shown in Table 2. Downhill treadmill exercise, $53,54,56$ ) single or repeated bouts of lengthening contraction, ${ }^{61,62)}$ and electrical stimulation ${ }^{55,57)}$ have been utilized as experimental models for eccentric exercise interventions.

Da Rocha et al. ${ }^{54)}$ reported that overtraining (OT) protocolsdownhill running-based overtraining (OTR/down), uphill running-based overtraining (OTR/up), and overtraining without inclination (OTR) - tended to induce a proinflammatory state and lead to skeletal muscle tissue injury. Indeed, proinflammatory cytokines were more sensitive to the OTR/down than to the OTR/ up or OTR. Hyldahl et al. ${ }^{63)}$ reported significant changes in the levels of two cytokines (monocyte chemoattractant protein-1 [MCP-1] and interferon $\gamma$-induced protein $10 \mathrm{kDa}$ [IP-10]) following eccentric contraction, indicating a potential role for IP-10 as a mediator of satellite cell activity and muscle regeneration. Overall, proinflammatory responses suggesting muscle damage or remodeling were upregulated following eccentric but not concentric contraction. Furthermore, muscle damage induced by eccentric exercise acutely upregulated proinflammatory cytokines (e.g., 


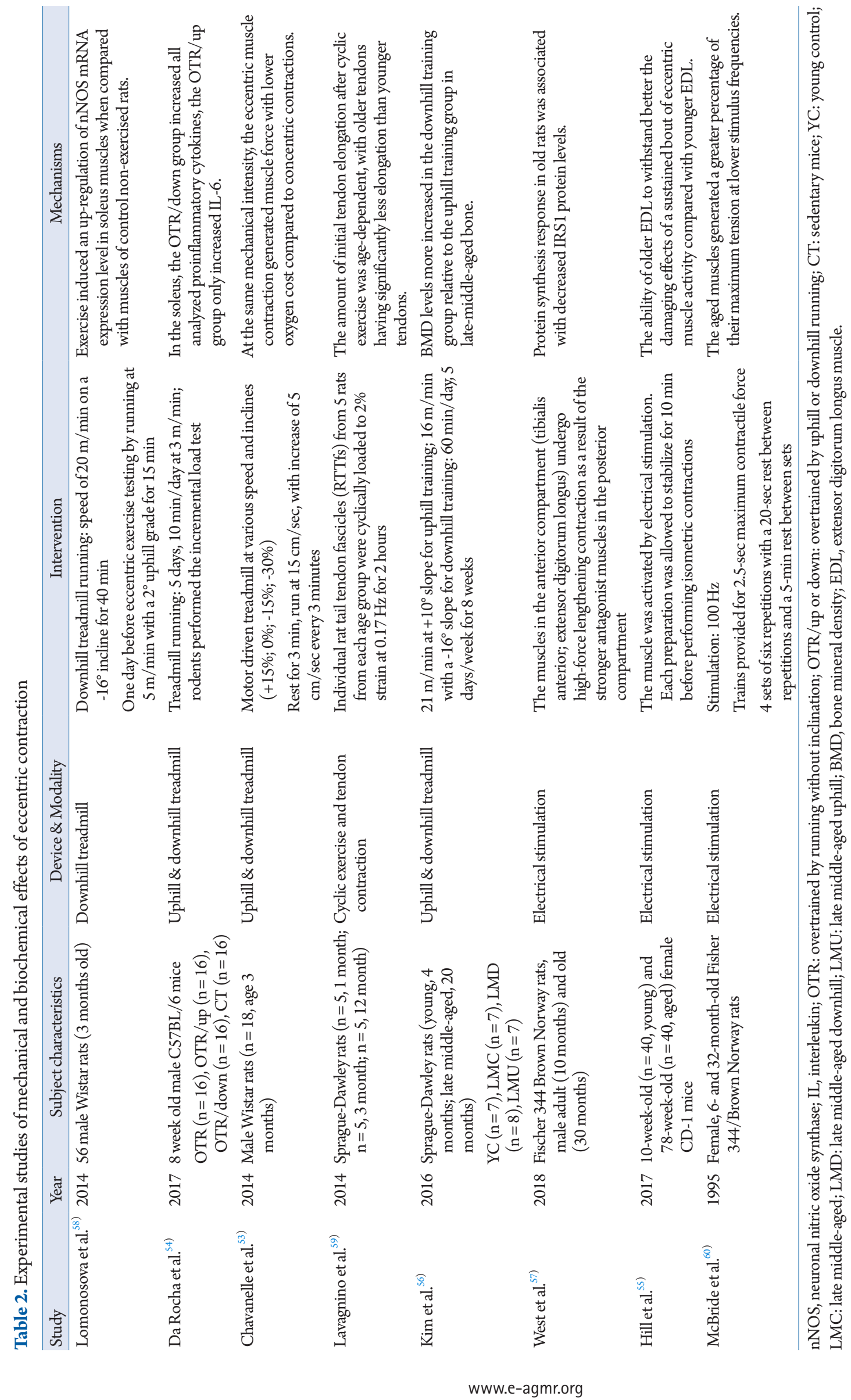


increased interleukin-6 [IL-6]) and, over a longer term, downregulated proinflammatory cytokines and upregulated anti-inflammatory cytokines (e.g., increased IL-10). ${ }^{64)}$

Satellite cell activity is important for skeletal muscle health throughout the human lifespan. Eccentric but not concentric contractions are associated with a transient damage response, the repair of which appears to be a function of satellite cells. Moreover, eccentric contractions promoted a greater satellite cell response than concentric contractions. ${ }^{63)}$ Eccentric exercise also reportedly upregulated muscle-specific genes associated with muscle cell hypertrophy. ${ }^{61,65)}$ Thus, eccentric muscle contraction is useful when a high degree of muscle strength is required, as it can stimulate satellite cell activity and boost anabolic signaling. ${ }^{28,66)}$

Kim et al. ${ }^{56)}$ reported that the rectus femoris (RF) but not vastus lateralis (VL) muscle mass was significantly increased in the downhill training group and that mTOR and MyoD expression in the RF muscle was likely significantly increased by downhill running exercise. Therefore, eccentric exercise may be suitable for improving muscle growth, repair, and remodeling. In this context, West et al. ${ }^{57)}$ observed that protein synthesis in old muscle was directed toward repairing muscle damage rather than synthesizing myofibrillar proteins that would increase muscle size and strength under electrical stimulation. In addition, one in-vitro study assessing muscle activity under electrical stimulation reported better responses to the damaging effects of a sustained bout of eccentric muscle activity in older extensor digitorum longus muscle (EDL) compared to younger EDLs. ${ }^{55)}$ Collectively, the results of these studies demonstrated the beneficial effects of eccentric exercise in inducing skeletal muscle growth and enhancing skeletal muscle function in aged groups.

Eccentric muscle contraction generates muscle force with a lower metabolic demand (e.g., lower oxygen cost) for the same mechanical intensity as concentric contraction. ${ }^{53)}$ At the muscle cell level, the reduced energy cost of eccentric muscle contraction compared to isometric and concentric contractions has been explained based on the cross-bridge theory. Huxley ${ }^{67)}$ found that cross-bridges were not necessarily tied to the hydrolysis of one adenosine triphosphate (ATP) per cross-bridge cycle (as is typically assumed for isometric and concentric contractions); rather, one ATP molecule was hydrolyzed per multiple cross-bridge cycles in eccentric contractions. However, some have argued that the higher force and lower energy cost of eccentric contractions are difficult to fit into the cross-bridge theory. Unmeasurable assumptions regarding cross-bridge properties, such as stiffness, duty ratio, and energy states, are required to estimate the energy storage capabilities of cross-bridges during muscle contraction. Therefore, other theories have been proposed to account for muscle properties during eccentric contraction, such as residual force enhancement, the long-lasting increase in force that persists after stretching the active muscle; nonuniform sarcomere length; and titin-actin interactions. $^{68)}$

Eccentric exercise can cause substantial changes in intramuscular structures, such as muscle fascicle length. ${ }^{69)}$ Lengthening contractions can increase muscle fascicle length, which decline with immobilization and can be increased through regular eccentric exercises. ${ }^{18)}$ Furthermore, eccentric loading can increase the number of sarcomeres in muscle fibers. ${ }^{47,48}$ Butterfield et al. ${ }^{48)}$ reported significantly increased sarcomere number in the vastus intermedius of rats after 10 days of downhill walking training. The existence of the sarcomeric protein myotilin was suggested to play a role in the development of new sarcomere structures after stretching stimulation. ${ }^{70)}$

\section{SAFETY AND ADVERSE EFFECTS}

As eccentric contractions have traditionally been associated with muscle damage, eccentric exercise has routinely been discouraged. However, some evidence indicates that eccentric exercise can be safely and effectively performed in older adults. Mueller et al. ${ }^{33)}$ and Sanudo et al. ${ }^{35)}$ suggested that muscle damage can be avoided by carefully increasing the muscle load over repeated exercise sessions beginning with a very low eccentric load- $30 \mathrm{~W}$ and $50 \mathrm{~W}$ for women and men, respectively; ${ }^{33)}$ first 3 weeks using one flywheel with a moment inertia of $0.025 \mathrm{~kg} / \mathrm{m}^{2}$ and after week 4 , a flywheel with a moment inertia of $0.05 \mathrm{~kg} / \mathrm{m}^{2} .^{35)}$ LaStayo et al. ${ }^{71,72)}$ reported that damage is inevitable when high eccentric forces are generated in muscles naive to eccentric contractions. However, no symptoms of damage, inflammation, or soreness occurred if the magnitude and duration of the force production were increased gradually.

In older adults with disease or those recovering from surgery, a phased progression of non-painful, non-injurious eccentric exercises is universally feasible owing to the relatively low exertion required. ${ }^{73)}$ In addition, 1:1 supervision and familiarization using progressively increased effort and load are recommended to ensure good form, comfort, and consistent performance and to reduce injury risk. Additionally, fall risk in older adults during stair descent, peak heart rate, systolic blood pressure, cardiac index, and expired ventilation were lower during eccentric exercise of equivalent volume to those of concentric exercise. ${ }^{73,74)}$ Eccentric exercise is currently performed to progressively increase the duration, frequency, and intensity of the exercise sessions and minimize the symptoms of damage. ${ }^{75)}$ Thus, eccentric exercise interventions are considered safe and suitable alternatives to traditional 
resistance exercises.

However, the level of intensity, the method of load progression, and the mode of delivery of eccentric exercise have been less studied compared to those in traditional resistance exercises, such as isometric or concentric contraction. ${ }^{76)}$ In particular, little is known about the specific protocols for eccentric exercise in aging muscles that are more susceptible to injury. Therefore, further studies are needed to develop a program that optimizes the intensity, duration, and modes of eccentric training while minimizing adverse effects in older adults.

\section{CONCLUSION}

Studies have been conducted in the last decade to elucidate the effects of eccentric contraction on aging muscles in older adults. We reviewed relevant literature on the use of the eccentric mode and its application, in which eccentric exercise has gained a great deal of interest across many fields. In addition, experimental studies can be used to reveal its mechanisms and benefits. The main finding of this review was that eccentric exercise is more effective than other traditional resistance exercises in improving muscle strength and mobility function in older adults. In particular, eccentric exercises using a specific device may more practically enable pure eccentric contractions. Eccentric exercise produces relatively greater force with lower energy consumption, making it an attractive option in strength programs to improve physical performance in older adults. However, continued research on appropriate protocols for eccentric contraction is needed to minimize adverse effects in this population.

\section{ACKNOWLEDGMENTS}

\section{CONFLICT OF INTEREST}

The researchers claim no conflicts of interest.

\section{FUNDING}

This study was supported by (the Rehabilitation Research \& Development Support Program NRCRSP-EX20010), National Rehabilitation Center, Ministry of Health and Welfare and the National Research Foundation of Korea (NRF) grant funded by the Korea government (MSIP) (No. 800-20200179).

\section{REFERENCES}

1. Aagaard P, Suetta C, Caserotti P, Magnusson SP, Kjaer M. Role of the nervous system in sarcopenia and muscle atrophy with aging: strength training as a countermeasure. Scand J Med Sci
Sports 2010;20:49-64.

2. Kalyani RR, Corriere M, Ferrucci L. Age-related and disease-related muscle loss: the effect of diabetes, obesity, and other diseases. Lancet Diabetes Endocrinol 2014;2:819-29.

3. Walston J, Fried LP. Frailty and the older man. Med Clin North Am 1999;83:1173-94.

4. Jang HC. Diabetes and muscle dysfunction in older adults. Ann Geriatr Med Res 2019;23:160-4.

5. Gault ML, Willems ME. Aging, functional capacity and eccentric exercise training. Aging Dis 2013;4:351-63.

6. Hortobagyi T, DeVita P. Favorable neuromuscular and cardiovascular responses to 7 days of exercise with an eccentric overload in elderly women. J Gerontol A Biol Sci Med Sci 2000; 55:B401-10.

7. LaStayo PC, WoolfJM, Lewek MD, Snyder-Mackler L, Reich T, Lindstedt SL. Eccentric muscle contractions: their contribution to injury, prevention, rehabilitation, and sport. J Orthop Sports Phys Ther 2003;33:557-71.

8. Lim JY. Therapeutic potential of eccentric exercises for age-related muscle atrophy. Integr Med Res 2016;5:176-81.

9. Lindstedt SL, LaStayo PC, Reich TE. When active muscles lengthen: properties and consequences of eccentric contractions. News Physiol Sci 2001;16:256-61.

10. Hessel AL, Lindstedt SL, Nishikawa KC. Physiological mechanisms of eccentric contraction and its applications: a role for the giant titin protein. Front Physiol 2017;8:70.

11. Lovering RM, Brooks SV. Eccentric exercise in aging and diseased skeletal muscle: good or bad? J Appl Physiol (1985) 2014; 116:1439-45.

12. Lee MY, Jung WS, Lee MG. Effects of a 12-week circuit training on fall-related fitness in elderly women with sarcopenia. Korean J Sports Sci 2017;26:1123-35.

13. Waters DL, Baumgartner RN, Garry PJ. Sarcopenia: current perspectives. J Nutr Health Aging 2000;4:133-9.

14. Doherty TJ. Invited review: aging and sarcopenia. J Appl Physiol (1985) 2003;95:1717-27.

15. Afilalo J, Karunananthan S, Eisenberg MJ, Alexander KP, Bergman $\mathrm{H}$. Role of frailty in patients with cardiovascular disease. Am J Cardiol 2009; 103:1616-21.

16. Manton KG, Stallard E, Corder L. Changes in the age dependence of mortality and disability: cohort and other determinants. Demography 1997;34:135-57.

17. Kim YH, Kim KI, Paik NJ, Kim KW, Jang HC, Lim JY. Muscle strength: a better index of low physical performance than muscle mass in older adults. Geriatr Gerontol Int 2016;16:577-85.

18. Raj IS, Bird SR, Shield AJ. Aging and the force-velocity relationship of muscles. Exp Gerontol 2010;45:81-90. 
19. Carlson BM, Dedkov EI, Borisov AB, Faulkner JA. Skeletal muscle regeneration in very old rats. J Gerontol A Biol Sci Med Sci 2001;56:B224-33.

20. Nilwik R, Snijders T, Leenders M, Groen BB, van Kranenburg J, Verdijk LB, et al. The decline in skeletal muscle mass with aging is mainly attributed to a reduction in type II muscle fiber size. Exp Gerontol 2013;48:492-8.

21. Choi SJ. Age-related functional changes and susceptibility to eccentric contraction-induced damage in skeletal muscle cell. Integr Med Res 2016;5:171-5.

22. Ochala J, Frontera WR, Dorer DJ, Van Hoecke J, Krivickas LS. Single skeletal muscle fiber elastic and contractile characteristics in young and older men. J Gerontol A Biol Sci Med Sci 2007; 62:375-81.

23. Lim JY, Choi SJ, Widrick JJ, Phillips EM, Frontera WR. Passive force and viscoelastic properties of single fibers in human aging muscles. Eur J Appl Physiol 2019;119:2339-48.

24. Frontera WR, Suh D, Krivickas LS, Hughes VA, Goldstein R, Roubenoff R. Skeletal muscle fiber quality in older men and women. Am J Physiol Cell Physiol 2000;279:C611-8.

25. Larsson L, Li X, Frontera WR. Effects of aging on shortening velocity and myosin isoform composition in single human skeletal muscle cells. Am J Physiol 1997;272(2 Pt 1):C638-49.

26. Waters DL. Intermuscular adipose tissue: a brief review of etiology, association with physical function and weight loss in older adults. Ann Geriatr Med Res 2019;23:3-8.

27. Oh SL, Kim DY, Bae JH, Lim JY. Effects of rural community-based integrated exercise and health education programs on the mobility function of older adults with knee osteoarthritis. Aging Clin Exp Res 2021;33:3005-14.

28. Hody S, Croisier JL, Bury T, Rogister B, Leprince P. Eccentric muscle contractions: risks and benefits. Front Physiol 2019; 10:536.

29. Tieland M, Trouwborst I, Clark BC. Skeletal muscle performance and ageing.J Cachexia Sarcopenia Muscle 2018;9:3-19.

30. Raj IS, Bird SR, Westfold BA, Shield AJ. Determining criteria to predict repeatability of performance in older adults: using coefficients of variation for strength and functional measures. J Aging Phys Act 2017;25:94-8.

31. Kim DY, Oh SL, Song W, Lim JY. Effects of lower extremity eccentric-based training on muscle strength and physical function in older adults: a randomized controlled pilot trial. Exerc Sci 2019;28:346-54.

32. LaStayo P, Marcus R, Dibble L, Wong B, Pepper G. Eccentric versus traditional resistance exercise for older adult fallers in the community: a randomized trial within a multi-component fall reduction program. BMC Geriatr 2017;17:149.
33. Mueller M, Breil FA, Vogt M, Steiner R, Lippuner K, Popp A, et al. Different response to eccentric and concentric training in older men and women. Eur J Appl Physiol 2009;107:145-53.

34. Raj IS, Bird SR, Westfold BA, Shield AJ. Effects of eccentrically biased versus conventional weight training in older adults. Med Sci Sports Exerc 2012;44:1167-76.

35. Sanudo B, Gonzalez-Navarrete A, Alvarez-Barbosa F, de Hoyo M, Del Pozo J, Rogers ME. Effect of flywheel resistance training on balance performance in older adults: a randomized controlled trial. J Sports Sci Med 2019; 18:344-50.

36. Dias CP, Toscan R, de Camargo M, Pereira EP, Griebler N, Baroni BM, et al. Effects of eccentric-focused and conventional resistance training on strength and functional capacity of older adults. Age (Dordr) 2015;37:99.

37. Gluchowski A, Dulson D, Merien F, Plank L, Harris N. Comparing the effects of two distinct eccentric modalities to traditional resistance training in resistance trained, higher functioning older adults. Exp Gerontol 2017;98:224-9.

38. Leszczak TJ, Olson JM, Stafford J, Brezzo RD. Early adaptations to eccentric and high-velocity training on strength and functional performance in community-dwelling older adults. J Strength Cond Res 2013;27:442-8.

39. Theodorou AA, Panayiotou G, Paschalis V, Nikolaidis MG, Kyparos A, Mademli L, et al. Stair descending exercise increases muscle strength in elderly males with chronic heart failure. BMC Res Notes 2013;6:87.

40. Katsura Y, Takeda N, Hara T, Takahashi S, Nosaka K. Comparison between eccentric and concentric resistance exercise training without equipment for changes in muscle strength and functional fitness of older adults. Eur J Appl Physiol 2019;119:158190.

41. Chen TC, Tseng WC, Huang GL, Chen HL, Tseng KW, Nosaka $\mathrm{K}$. Superior effects of eccentric to concentric knee extensor resistance training on physical fitness, insulin sensitivity and lipid profiles of elderly men. Front Physiol 2017;8:209.

42. Roig M, O’Brien K, Kirk G, Murray R, McKinnon P, Shadgan B, et al. The effects of eccentric versus concentric resistance training on muscle strength and mass in healthy adults: a systematic review with meta-analysis. BrJ Sports Med 2009;43:556-68.

43. Isner-Horobeti ME, Dufour SP, Vautravers P, Geny B, Coudeyre E, Richard R. Eccentric exercise training: modalities, applications and perspectives. Sports Med 2013;43:483-512.

44. Vogt M, Hoppeler HH. Eccentric exercise: mechanisms and effects when used as training regime or training adjunct. J Appl Physiol (1985) 2014;116:1446-54.

45. Roig M, Macintyre DL, Eng JJ, Narici MV, Maganaris CN, Reid WD. Preservation of eccentric strength in older adults: evidence, 
mechanisms and implications for training and rehabilitation. Exp Gerontol 2010;45:400-9.

46. Fenwick AJ, Wood AM, Tanner BC. Effects of cross-bridge compliance on the force-velocity relationship and muscle power output. PLoS One 2017; 12:e0190335.

47. Butterfield TA. Eccentric exercise in vivo: strain-induced muscle damage and adaptation in a stable system. Exerc Sport Sci Rev 2010;38:51-60.

48. Butterfield TA, Leonard TR, Herzog W. Differential serial sarcomere number adaptations in knee extensor muscles of rats is contraction type dependent. J Appl Physiol (1985) 2005;99: 1352-8.

49. Lavender AP, Nosaka K. Comparison between old and young men for changes in makers of muscle damage following voluntary eccentric exercise of the elbow flexors. Appl Physiol Nutr Metab 2006;31:218-25.

50. Guilhem G, Cornu C, Nordez A, Guevel A. A new device to study isoload eccentric exercise. J Strength Cond Res 2010; 24:3476-83.

51. Vikne H, Refsnes PE, Ekmark M, Medbo JI, Gundersen V, Gundersen K. Muscular performance after concentric and eccentric exercise in trained men. Med Sci Sports Exerc 2006;38:1770-81.

52. Paddon-Jones D, Leveritt M, Lonergan A, Abernethy P. Adaptation to chronic eccentric exercise in humans: the influence of contraction velocity. Eur J Appl Physiol 2001;85:466-71.

53. Chavanelle V, Sirvent P, Ennequin G, Caillaud K, Montaurier C, Morio B, et al. Comparison of oxygen consumption in rats during uphill (concentric) and downhill (eccentric) treadmill exercise tests. J Sports Sci Med 2014;13:689-94.

54. Da Rocha AL, Pereira BC, Teixeira GR, Pinto AP, Frantz FG, Elias LL, et al. Treadmill slope modulates inflammation, fiber type composition, androgen, and glucocorticoid receptors in the skeletal muscle of overtrained mice. Front Immunol 2017;8: 1378.

55. Hill C, James RS, Cox VM, Tallis J. The effect of increasing age on the concentric and eccentric contractile properties of isolated mouse soleus and extensor digitorum longus muscles. J Gerontol A Biol Sci Med Sci 2018;73:579-87.

56. Kim JS, Lee YH, Yi HK. Gradual downhill running improves age-related skeletal muscle and bone weakness: implication of autophagy and bone morphogenetic proteins. Exp Physiol 2016; 101:1528-40.

57. West DW, Marcotte GR, Chason CM, Juo N, Baehr LM, Bodine $\mathrm{SC}$, et al. Normal ribosomal biogenesis but shortened protein synthetic response to acute eccentric resistance exercise in old skeletal muscle. Front Physiol 2019;9:1915.

58. Lomonosova YN, Shenkman BS, Kalamkarov GR, Kostromino- va TY, Nemirovskaya TL. L-arginine supplementation protects exercise performance and structural integrity of muscle fibers after a single bout of eccentric exercise in rats. PLoS One 2014; 9:e94448.

59. Lavagnino M, Bedi A, Walsh CP, Sibilsky Enselman ER, Sheibani-Rad S, Arnoczky SP. Tendon contraction after cyclic elongation is an agedependent phenomenon: in vitro and in vivo comparisons. Am J Sports Med 2014;42:1471-7.

60. McBride TA, Gorin FA, Carlsen RC. Prolonged recovery and reduced adaptation in aged rat muscle following eccentric exercise. Mech Ageing Dev 1995;83:185-200.

61. Barash IA, Mathew L, Ryan AF, Chen J, Lieber RL. Rapid muscle-specific gene expression changes after a single bout of eccentric contractions in the mouse. Am J Physiol Cell Physiol 2004;286:C355-64.

62. Marqueste T, Giannesini B, Fur YL, Cozzone PJ, Bendahan D. Comparative MRI analysis of T2 changes associated with single and repeated bouts of downhill running leading to eccentric-induced muscle damage. J Appl Physiol (1985) 2008;105:299307.

63. Hyldahl RD, Olson T, Welling T, Groscost L, Parcell AC. Satellite cell activity is differentially affected by contraction mode in human muscle following a work-matched bout of exercise. Front Physiol 2014;5:485.

64. Paulsen G, Mikkelsen UR, Raastad T, Peake JM. Leucocytes, cytokines and satellite cells: what role do they play in muscle damage and regeneration following eccentric exercise? Exerc Immunol Rev 2012;18:42-97.

65. Douglas J, Pearson S, Ross A, McGuigan M. Chronic adaptations to eccentric training: a systematic review. Sports Med 2017;47: 917-41.

66. Franchi MV, Reeves ND, Narici MV. Skeletal muscle remodeling in response to eccentric vs. concentric loading: morphological, molecular, and metabolic adaptations. Front Physiol 2017; 8:447.

67. Huxley AF. Muscle structure and theories of contraction. Prog Biophys Biophys Chem 1957;7:255-318.

68. Nishikawa KC, Lindstedt SL, LaStayo PC. Basic science and clinical use of eccentric contractions: history and uncertainties. J Sport Health Sci 2018;7:265-74.

69. Reeves ND, Maganaris CN, Longo S, Narici MV. Differential adaptations to eccentric versus conventional resistance training in older humans. Exp Physiol 2009;94:825-33.

70. Carlsson L, Yu JG, Moza M, Carpen O, Thornell LE. Myotilin: a prominent marker of myofibrillar remodelling. Neuromuscul Disord 2007; 17:61-8.

71. LaStayo PC, Ewy GA, Pierotti DD, Johns RK, Lindstedt S. The 
positive effects of negative work: increased muscle strength and decreased fall risk in a frail elderly population. J Gerontol A Biol Sci Med Sci 2003;58:M419-24.

72. Lastayo PC, Reich TE, Urquhart M, Hoppeler H, Lindstedt SL. Chronic eccentric exercise: improvements in muscle strength can occur with little demand for oxygen. Am J Physiol 1999; 276:R611-5.

73. Vallejo AF, Schroeder ET, Zheng L, Jensky NE, Sattler FR. Cardiopulmonary responses to eccentric and concentric resistance exercise in older adults. Age Ageing 2006;35:291-7.

74. Laroche D, Joussain C, Espagnac C, Morisset C, Tordi N,
Gremeaux V, et al. Is it possible to individualize intensity of eccentric cycling exercise from perceived exertion on concentric test? Arch Phys Med Rehabil 2013;94:1621-7.

75. Chen TC, Tseng WC, Huang GL, Chen HL, Tseng KW, Nosaka $\mathrm{K}$. Low-intensity eccentric contractions attenuate muscle damage induced by subsequent maximal eccentric exercise of the knee extensors in the elderly. Eur J Appl Physiol 2013;113:100515.

76. LaStayo P, Marcus R, Dibble L, Frajacomo F, Lindstedt S. Eccentric exercise in rehabilitation: safety, feasibility, and application.J Appl Physiol (1985) 2014;116:1426-34. 